\title{
Performance Improvement of DWT OFDM in Presence of Phase Noise Using LDPC Coding
}

\author{
Neethu S. $\mathbf{J}^{1}$, Ms. Sreedevi $\mathrm{P}^{2}$. \\ ${ }^{1,2}$ Dept. of Electronics and Communication Engineering MBCET, Trivandrum, India
}

\begin{abstract}
Ofdm Is A Multicarrier Transmission System Which Is Used In Many Modern And Emerging Communication Standards Such As Dvb, Wireless Local Area Networks Etc. The Conventional Ofdm System Uses Fft As Their Basic Building Block. The Disadvantages Of Fft Ofdm Are The Addition Of Cyclic Prefixes Between The Carriers Which Consumes More Bandwidth. Our Proposed Work Makes Use Of The Dwt Principle To Generate Ofdm Which Improves The Bandwidth Without Cyclic Prefix. The System Is Designed, Especially Under The Presence Of Phase Noise To Study Its Effect. Ldpc Encoding Technique Is Used To Reduce The Phase Noise Of The Proposed System. Different Wavelet Transforms Performance Is Compared With Fft Ofdm. The Results Clearly Show That Dwt Excels Fft.

Index Terms: Ofdm, Fft, Dwt. Phase Noise, Ber, Ldpc.
\end{abstract}

\section{Introduction}

Orthogonal Frequency Division Multiplexing (OFDM) is the division of high bit streams to low bits and it is a method of encoding of digital data on multiple carrier frequencies. OFDM is combined with both modulation and multiplexing. Frequency Division Multiplexing (FDM) which divides the available bandwidth into different subcarriers but the subcarriers will not overlap with each other. In case of OFDM, the carriers are orthogonal to each other thus providing overlapping. The advantage of OFDM is it will provide high spectral efficiency. OFDM can be effectively implemented using Fast Fourier Transform (FFT).It is the most popular transform to obtain frequency spectrum of signals. Here, cyclic prefix are added to reduce the Inter Symbol Interferences (ISI). But the addition of cyclic prefixes reduces the spectrum efficiency. This is one of the disadvantages of FFT OFDM.

The disadvantage of FFT OFDM can be solved by using DWT OFDM [1]. The DWT OFDM divides the symbol into two components such as low and high. The low frequency components are approximated coefficients and high frequency components are detailed coefficients. Here there is no need for cyclic prefix thus bandwidth can be consumed. The two important properties of DWT are perfect reconstruction property and orthonormal basis property. With this property, we can perfectly reconstruct the symbol at the receiver. Here, wavelet transforms are considered in the presence of phase noise. Phase noise is a random process which is caused due to the fluctuations of the transmitter and receiver oscillators. The phase noise leads to leakage of signals which destroys the orthogonality among the carriers. The two effects of phase noise on OFDM are Common Phase Error (CPE) and Inter Carrier Interferences (ICI) [1]. CPE causes subcarrier rotation and ICI introduces interference to subcarriers. This paper proposes the improvement of BER in the presence of phase noise. For that LDPC coded DWT OFDM is performed in the presence of phase noise and the outputs are plotted.

\section{Dwt Ofdm}

The Discrete Wavelet Transform (DWT) [2] of a sequence having N components can be expressed as $\mathrm{N} \times \mathrm{N}$ matrix. In fourier analysis the signal can be divided into a set of sinusoidal functions. But wavelet transform decomposed the signal by a LPF and HPF after down sampling. From low pass filter we get approximation coefficient and from high pass filter we get detailed co-efficient. The approximated coefficients consist of more information. Wavelet OFDM is similar to FFT OFDM. Here we are using IDWT and DWT instead of IFFT and FFT. The block diagram shown in figure 2 will give more information about its working.

On the transmitted side the input data is modulated using QAM and then we applied IDWT. The output will be a sequence of OFDM signal by up sampling and filtering. The IDWT output can be given as,

$$
d(k)=\sum_{m=0}^{\infty} \cdot \sum_{n=0}^{\infty} D_{m}^{n} 2^{\frac{m}{2}} \varphi\left(2_{k}^{m}-n\right)
$$

Where, $D_{m}^{n}$ are wavelet coefficients and $\varphi(t)$ is the wavelet function. At the receiver reverse operation is carried out. The DWT dounsamples the received signal and the output can be written as,

$$
D_{m}^{n}=\sum_{k=0}^{N-1} d(k) 2^{\frac{m}{2}} \varphi\left(2_{k}^{m}-n\right)
$$


The two important properties of DWT OFDM are orthonormality and perfect reconstruction property. The LPF and HPF have to satisfy orthonormal to satisfy orthonormal bases which means they must be orthogonal and normal to each other. The possible ways are,

$$
\begin{aligned}
& <g \cdot g^{*}>=1 \\
& <h \cdot h^{*}>=1 \\
& <g \cdot h^{*}>=0 \\
& <h \cdot g^{*}>=0
\end{aligned}
$$

Where, (3) or (4) related to normal property and (5) or (6) related to orthogonal property.The block diagram of wavelet based ofdm can be given as,

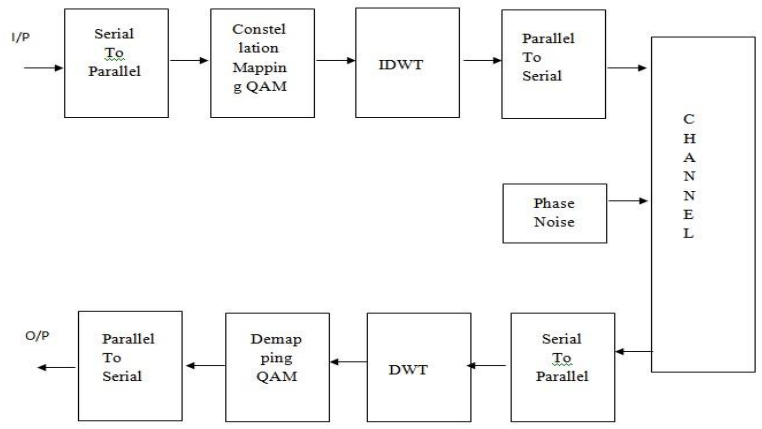

Fig .1.Block diagram of DWT OFDM

The IDFT used in transmitter side contains two or three filter banks. The receiver reproduces the approximated coefficients which are modulated carriers and detailed coefficients which are zeros then discarded. The IDWT transmitter and receiver can be drawn as follows,
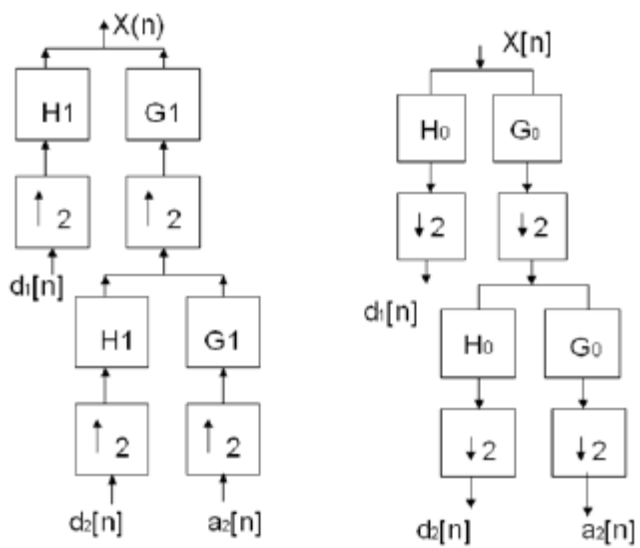

IDWT used in DWT used in transmitter side receiver side

Fig.2. IDWT/DWT in Transmitter/Receiver

Modulated data carriers can be given as $a_{n}$ and for $z_{n}$ zero padding will done. Then series of upsampling will be done. This converts the carriers required for final transmission version $X_{n}$. The other applications of wavelets than OFDM are computer and human vision, finger print compressions, denoising noisy data, musical tones etc.

\section{Phase Noise In Ofdm}

Phase noise is the random noise which is occurred at transmitter or receiver oscillators. It is one of the important disadvantages faced by the OFDM signal. Theoretically phase noise in OFDM can be modelled as Brownian process. An OFDM signal affected with phase noise will resulting in two types of phase errors at the receiver side such as Common Phase Error (CPE) and Inter Carrier Interference (ICI). The received signal with phase error can be expressed as [1], 


$$
y(k)=S_{k}+e_{k}
$$

$e_{k}$ is the phase error and is given by,

$$
e_{k}=\frac{j}{N} \sum_{r=0}^{N-1} S_{r} \sum_{n=0}^{N-1} \emptyset(n) e^{j\left(\frac{2 \pi}{N}\right)(r-k) n}
$$

For $\mathrm{r}=\mathrm{k}$ the above equation becomes,

$$
e_{k}=j \cdot S_{k} \cdot \varnothing
$$

Known as Common Phase Error (CPE) and $\emptyset$ is the angle due to the rotation of the constellation is given by,

$$
\emptyset=\frac{1}{N} \sum_{n=0}^{N-1} \emptyset(n)
$$

If $r \neq k$ equation (10) will be the Inter Carrier Inter Interference (ICI).

The effects of the CPE and the ICI in the OFDM signal may be given as,

1. CPE leads to the rotation of the constellation which needs to be corrected at the receiver side. But it will difficult if the number of subcarriers are large.

2. ICI makes the system to lose its orthogonality and resulting in severe ruining of the received signal.

3. The overall effect of these CPE and ICI can be made the

4. degradation of the BER performance of the system.

Therefore, in this paper we are trying to improve the BER performance using different wavelet transforms such as haar and $\mathrm{db} 2$ in presence of phase noise. Also to improve the performance of the system, the effect of phase noise should be reduced. For that a coding technique which is LDPC (Low Density Parity Check) codes are used. The encoded data is transmitted in presence of phase noise and it is decoded at the receiver end will improve the BER performance.

\section{Proposed Work}

The proposed work used LDPC coding [3] technique to encode and decode the data and DWT OFDM in presence of phase noise. Figure 3 represents the proposed block diagram. That is the LDPC encoded data is used for taking IDWT and phase noise is added at the channel. At the receiver side LDPC decoding will be done after taking DWT.

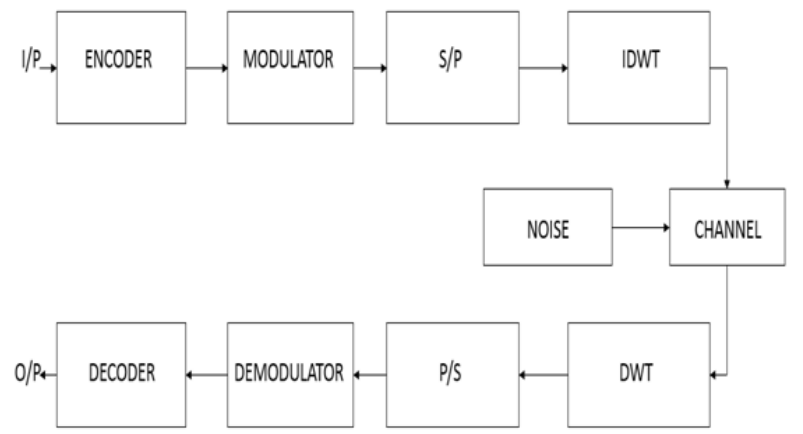

Fig.3. Proposed DWT Block diagram

For an LDPC encoding an LDPC matrix along with a parity check matrix is created. The check matrix can be represented by using a tanner graph. An example for a check matrix with dimension $4 \times 8$ is given as,

$$
H=\left[\begin{array}{llllllll}
0 & 1 & 0 & 1 & 1 & 0 & 0 & 1 \\
1 & 1 & 1 & 0 & 0 & 1 & 0 & 0 \\
0 & 0 & 1 & 0 & 0 & 1 & 1 & 1 \\
1 & 0 & 0 & 1 & 1 & 0 & 1 & 0
\end{array}\right]
$$


By using the check matrix, check vector will be generated. Along with the check vector data is transmitted. The check vector and the data bits can be said as the encoded message bits. This encoded signal is the message signal which is transmitted. The tanner graph for the above matrix can be drawn as,

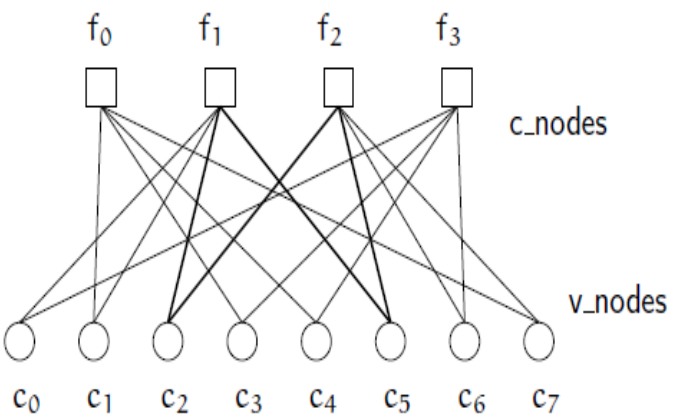

Fig.4. Tanner Graph

Here, the number of rows can be arranged as check nodes(c nodes) and the number of columns can be arranged as variable nodes ( $\mathrm{v}$ nodes). At the receiver side after DWT the symbols will be decoded. In the decoding section the received bits will check the parity by using the paths denoted in the tanner graph. The obtained output will have better BER performance when compared with the existing methods.

\section{Simulation Results}

The BER of DWT OFDM in the absence of phase noise is plotted in figure 5.

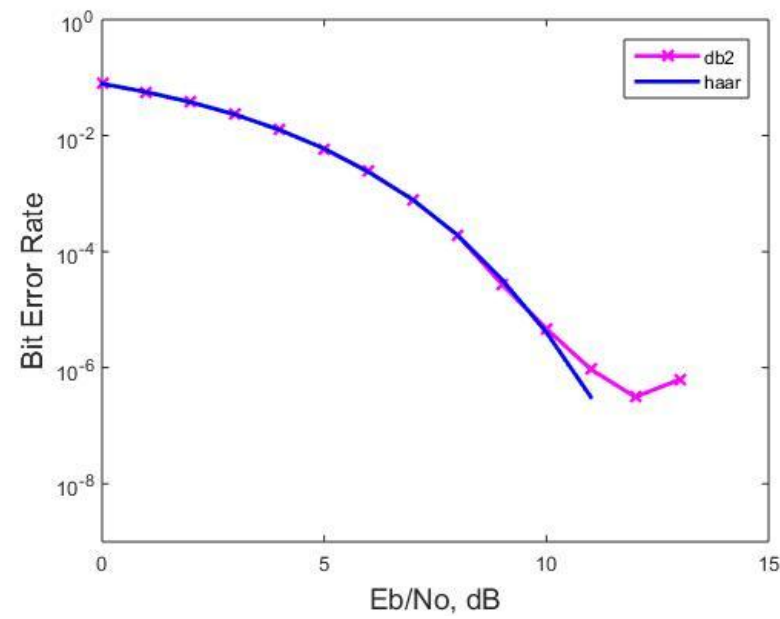

Fig. 5.BER performance of Haar and $\mathrm{db} 2$ without adding Phase noise

For doing a comparison BER of FFT OFDM in the absence of phase noise is also done.The BER performance of FFT OFDM, DWT OFDM ( Haar and Daubchies) can be plotted as shown in figure below.

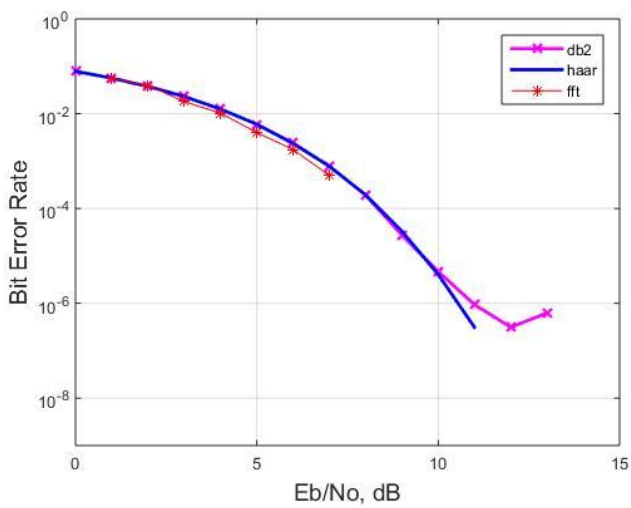

Fig.6. BER performance of FFT, DWT (Haar and db2) without adding phase noise 
From the above simulation result it is clear that in the absence of phase noise both the transforms has almost same BER performance. While adding phase noise the simulation results are as shown in figure 7 . The BER performance of FFT OFDM, Haar wavelet transform and Daubchies wavelet transform in the presence of Phase noise were plotted. While adding phase noise DWT OFDM is having better performance when compared with FFT.

To reduce the effect of phase noise an error correction code LDPC (Low Density Parity Check) is used. That is the input is encoded before transmission and it is decoded at the receiver. Since LDPC's are error correction codes used in noisy environments, the effect of phase noise added in DWT OFDM should reduced and the Bit Error Rate performance should be better compared with non coded outputs. The BER of Haar transforms after LDPC coding and the theoretical simulation of a normal OFDM symbol is plotted in figure 8.

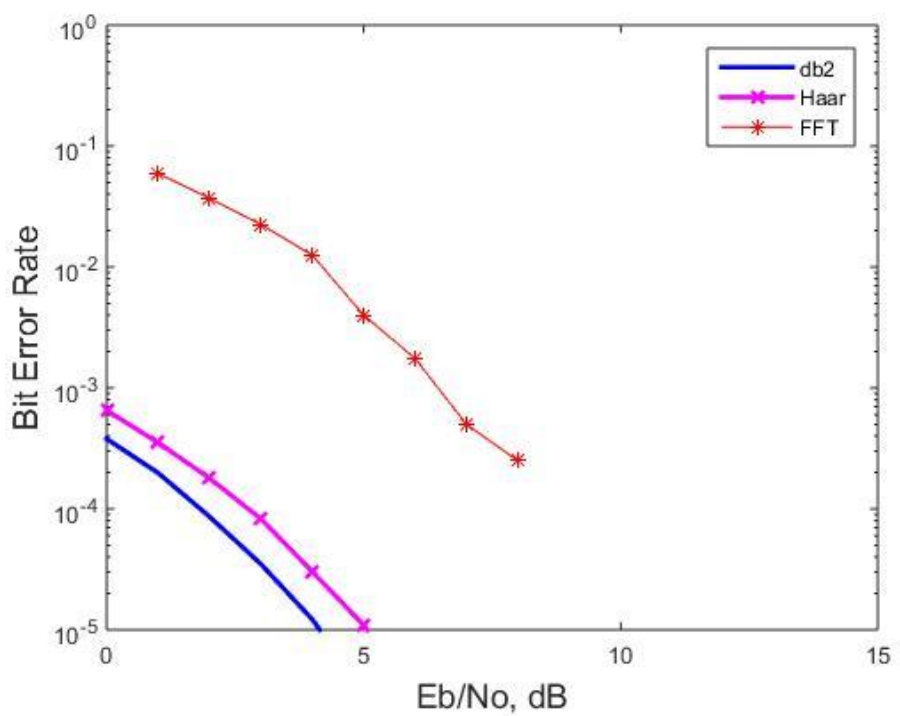

Fig.7. BER performance of FFT, Haar and $\mathrm{db} 2$ in presence of phase noise.

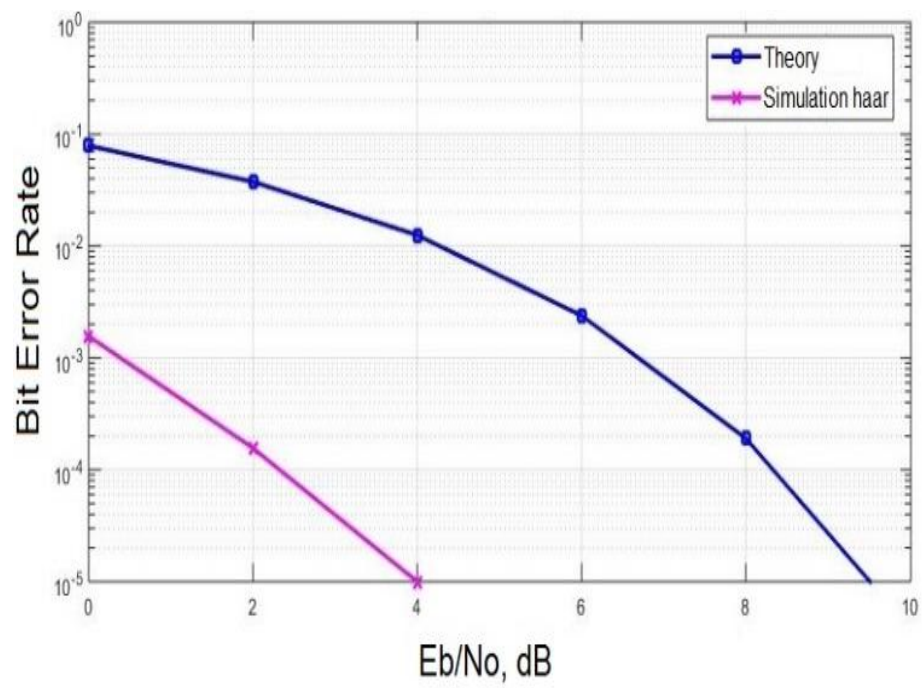

Fig.8. BER performance of Haar after LDPC coding

\section{Conclusion}

The disadvantage of FFT OFDM which is the addition of cyclic prefix can be overcome by using DWT OFDM. Thus the bandwidth can be reduced. The DWT OFDM is performed in presence of phase noise which is a major factor that affects OFDM symbols. For doing comparison, first the BER of FFT OFDM and DWT OFDM without adding phase noise are performed separately. From the results, DWT and FFT OFDM have almost same BER. In presence of phase noise for an SNR $=2 \mathrm{db}$ FFT has BER of $10^{-1}$ while Haar and Daubchies wavelet transform has BER of $10^{-3}$ and near to $10^{-4}$ respectively. In order to improve the BER performance by removing phase noise, LDPC coding is done. After LDPC coding, for SNR $=2 \mathrm{db}$ Haar achieved a BER of $10^{-4}$ which is better than the non coded output. 


\section{References}

[1] N. Hariprasad and G. Sundari,"Comparative Analysis of the BER Performance of DWT OFDM over that of FFT OFDM in Presence of Phase Noise", International Conference on Robotics, Automation, Control and Embedded Systems - RACE 2015 18-20 February 2015, Hindustan University, Chennai, India.

[2] Amara Graps, "An Introduction to Wavelets" IEEE Computational Science and Engineering, Summer 1995, vol. 2, num. 2, published by the IEEE Computer Society, 10662 Los Vaqueros Circle, Los Alamitos, CA 90720, USA.

[3] Bernhard M.J. Leiner, 'LDPC Codes - a brief Tutorial', 2005.

[4] JKhaizuran Abdullah and Zahir M. Hussain, "Studies on DWT-OFDM and FFT-OFDM Systems" International conference Muscut Feb 15-19, 2009 (ICCCP'09) PP 383-386.

[5] Wu S. \& Bar-Ness. Y. (2004). OFDM systems in the presence of phase noise: Consequences and solutions. IEEE Transactions on communications 52. 1988-1996.

[6] Syed Mohammed Sajid, Lakshmi C R,'DCT Based Imroved OFDM Communication”, International Journal of Engineering and Innovative Technology (IJEIT) Volume 2, Issue 12, June 2013.

[7] Deepak Gupta, Vipin B Vats, Kamal K Garg,'Performance Analysis of DFT OFDM,DCT OFDM and DWT OFDM Systems in AWGN Channel", The Fourth International Conference on Wireless and Mobile Communications.

[8] S. K. Singh, N. Sood, and A. K. Sharma,' Performance Evaluation of LDPC and Turbocoded OFDM System in Nakagami-M Fading', International Journal of Computer Theory and Engineering, Vol. 4, No. 4, August 2012. 